\section{Das Lernen ist des Müllers Lust}

\author{
E. Taverna
}

Fortbildung ist weit mehr als nur Lernen. Die «Union Européenne des Médecins Spécialistes» UEMS definiert in der sogenannten "Basler Deklaration", was unter "continuing professional development" CPD zu verstehen ist. Das Papier richtet sich ausdrücklich an Patienten, Ärzte, ärztliche Vereinigungen, Angestellte des Gesundheitswesens, Geldgeber und Politiker. Warum das Thema Fortbildung eine so grosse Bedeutung erlangt hat, wird mit gesellschaftlichen Veränderungen begründet. Die Rolle des Arztes werde kontrovers diskutiert und ändere sich rasch, die Medizintechnik nehme an Bedeutung zu und die Finanzierung der Gesundheitssysteme sei immer schwieriger. In einer deregulierten Welt hätten sich die Ansprüche der Konsumenten an die Medizin gewandelt. Gefordert seien vermehrt Effizienz, Zuverlässigkeit, Offenheit und Transparenz. Die professionelle Erziehung durch den Zyklus von «Lernen Arbeiten - Lernen» fördere die Sicherheit und sei Teil eines umfassenden Sozialvertrages zwischen allen Partnern der Gesundheitsindustrie. Mehrmals wird aber auch darauf hingewiesen, wie wichtig eine klar geregelte Finanzierung ist.

\section{Die neue Lernkultur}

Alle Konzepte betonen die lange Tradition freiwilliger medizinischer Fortbildung. Doch besser als Vertrauen ist anscheinend Kontrolle. Was Kollegenkränzchen, Balintgruppen, Qualitätszirkel und Kurse anbieten, wird nach eurokompatiblen Kreditpunkten bewertet, registriert und kontrolliert. Der FMH-Entwurf für eine Fortbildungsordnung FBO entwickelt aus der erwähnten Basler Deklaration ein Programm, das Ziel und Zweck, Art und Umfang und die Erfüllungsbedingungen formuliert. Die permanente Fortbildung als ethische Pflicht zum planmässigen Lernen kommt vorläufig mit 10 Fortbildungstagen jährlich aus. Die Fachgesellschaften gewichten die Fortbildungskategorien für die benötigten 50 Credits, was ungefähr gleich viel Stunden entsprechen soll, wobei die Kantonalen Gesellschaften ihren Mitgliedern verbindliche Inhalte, zum Beispiel für den Notfalldienst, vorschreiben können. Über die Erfüllung der Fortbildungspflicht innerhalb der dreijährigen Kontrollperiode entscheidet als einzige Instanz die Fachgesellschaft. Wer sein Soll erfüllt hat, bekommt von der FMH alle drei Jahre ein Fortbildungsdiplom und einen entsprechenden Vermerk im Ärzteverzeichnis auf der FMH-Homepage. Nichterfüllung bedeutet den Verlust des FMH-Titels. Im Gegensatz zum europäischen Vorbild wird über die Finanzierung dieser CPD nichts ausgesagt.

Wissen und Können ist die eine Seite, Marketing und Ökonomie die andere. Die Standards der medizinischen Versorgung werden durch viele Akteure festgelegt. Das klingt schon fast wie eine Durchhalteparole, wenn es im Grundsatzartikel heisst: «Umfang und Inhalt der Fortbildung werden durch die Ärzteschaft bestimmt", denn diese Gemeinschaft zerbröselt immer schneller im zunehmenden Verdrängungskampf der viel zu vielen. Mit Qualität lässt sich vortrefflich gegenseitig mauern und zudem ist absehbar, dass die zukünftige Lehrmaschinerie die Spielregeln mitbestimmt. Die Anforderungen an diese Qualitäten werden zahlreich und widersprüchlich sein. Da klingt ein Satz wie: "Besonderes Gewicht ist der Entwicklung der ärztlichen Haltung als kontinuierlichem Prozess beizumessen" schon verdächtig nach kommendem Unheil.

\section{Die neuen Zwänge}

Chancen und Risiken liegen nahe beieinander. Das neue Bildungsideal vom lebenslangen Lernen klänge verheissungsvoll, wäre da nicht die stillschweigende Verknüpfung mit Wettbewerb und wirtschaftlicher Verwertung. Der aktuelle Bildungsbegriff fordert von den Schulen, sich auf die Bedürfnisse des Marktes auszurichten, und wer darin bestehen will, soll seine Berufsbiographie ständig neu- und weiterschreiben. Lebenslanges Lernen ist nicht eine freudig gewählte Option, sondern reine Überlebensstrategie. Der flexiblen Fachschule folgt die berufspolitische Regulierung durch einen vom einzelnen nicht mehr beeinflussbaren Kontrollapparat. Die besondere Hervorhebung der "Haltung" als ethisch-moralische Zielsetzung ruft nach normativen Schulungsprogrammen zur Integration in die erwünschte Ideologie. Es hat lange gedauert, bis die Erziehung der Mediziner vom patriarchalisch-autoritären Stil Abschied nahm. Aber welches sind die neuen Berufsbilder, die hinter den schönen Worten von der offenen, sich weiterentwickelnden und lebenslang lernenden Gesellschaft verborgen sind? Könnten es Fachidioten sein, lebenslänglich lern- und therapiebedürftige Modernisierungsopfer, die der permanenten, lückenlosen Führung und Kontrolle bedürfen? 